\title{
GENERALIZED THERMAL RESISTANCE
}

BY

J. A. LEWIS*

Bell Telephone Laboratories, Inc. Murray Hill, New Jersey

1. Introduction. The thermal resistance of a conducting body $V$ is usually defined (see, e.g., [3] $\left.{ }^{* *}\right)$ in terms of a harmonic function $u\left(x_{1}, x_{2}, x_{3}\right)$, satisfying the boundary conditions

$$
\begin{aligned}
u & = \begin{cases}u_{1}, & \text { on } S_{1}, \\
u_{0}, & \text { on } S_{0},\end{cases} \\
u_{, i} n_{i} & =0, \text { on } S_{a},
\end{aligned}
$$

where $S=S_{1}+S_{0}+S_{a}$ is the boundary of $V, n_{i}$ is the outward normal vector, and $u_{1}$ and $u_{0}$ are constants. We shall refer to $S_{1}$ as the inlet, $S_{0}$ as the outlet, and $S_{a}$ as the adiabatic surface of the body $V$.

The thermal resistance $R$ of this configuration, i.e., the body $V$ with given inlet and outlet, is then defined by the equation

$$
R(u)=\left(u_{1}-u_{0}\right) / Q(u),
$$

where

$$
Q(u)=\int_{S_{i}} u_{, i} n_{i} d S,
$$

the total transmitted heat power.

By applying Green's identity to Eq. (1.1), using the harmonicity of $u$ in $V$, we obtain the alternate forms

$$
R(u)=\left(u_{1}-u_{0}\right)^{2} / D(u)=D\left(q_{i}\right) / Q^{2}\left(q_{i}\right),
$$

where $q_{i}=u_{, i}, Q\left(q_{i}\right)=Q(u)$, and the Dirichlet integrals $D$ are given by

$$
D(u)=D\left(q_{i}\right)=\int_{V} q_{i} q_{i} d V=\int_{V} u_{, i} u_{, i} d V .
$$

If $u_{1}-u_{0}$ is interpreted as a potential difference and $Q$ as total current, Eqs. (1.1) and (1.2) also give the electrical resistance of the configuration for unit electrical conductivity. The two forms given by Eq. (1.2) are simply the familiar " $E^{2} / R$ ", " $I{ }^{2} R$ " relations between the power dissipation $D$, the potential drop $E=u_{1}-u_{0}$, and the total current, $I=Q(u)$.

Since $u_{1}-u_{0}$ is obviously the maximum temperature difference in the body $V, R(u)$ gives the maximum temperature difference for unit transmitted heat power and thus serves as a power rating for the body, at least for these boundary conditions. Unfortu-

*Received April 2, 1960.

**Numbers in square brackets refer to the bibliography. 
nately, in contrast to the problem of electrical conduction, in practice one encounters a wide variety of boundary conditions in heat flow problems. We shall show, in fact, that the temperature rise in general is greater for given input heat power than that given by the above Dirichlet conditions, so that $R(u)$ is unsafe to use as a power rating, unless, of course, it is certain that the above boundary conditions are satisfied.

Very often in practical problems the exact boundary conditions are unknown. In the following, we consider two other boundary value problems, which we regard as typical, namely the Neumann problem and the Robin-Neumann problem, both with piecewise constant boundary values. We extend the definition of thermal resistance to these cases in a natural and expeditious way and obtain upper and lower bounds, which include the resistance in all three problems between them and thus serve as universal bounds, at least for the class of problems considered.

2. The Neumann problem. The temperature $v$, again harmonic in $V$, now satisfies the boundary conditions

$$
v_{. i} n_{i}=\left\{\begin{array}{lll}
Q / S_{1}, & \text { on } & S_{1}, \\
0, & \text { on } & S_{a}, \\
-Q / S_{0}, & \text { on } & S_{0},
\end{array}\right.
$$

where $Q$ is the total input heat power. In this case the thermal resistance is not usually defined. We may extend the definition, however, by using the analogy with electrical conduction and requiring that the resistance $R(v)$ again satisfy the usual " $I^{2} R$ " relation between the power dissipation $D$ and the total current $Q$, namely

$$
D(v)=Q^{2} R(v) .
$$

If we apply Green's identity, we obtain

$$
R(v)=\left(\langle v\rangle_{1}-\langle v\rangle_{0}\right) / Q,
$$

where the average inlet and outlet temperatures $\langle v\rangle_{1}$ and $\langle v\rangle_{0}$ are given by

$$
\langle v\rangle_{1}=S_{1}^{-1} \int_{S_{1}} v d S, \quad\langle v\rangle_{0}=S_{0}^{-1} \int_{S_{0}} v d S .
$$

As before, we have the alternate forms

$$
R(v)=\left(\langle v\rangle_{1}-\langle v\rangle_{0}\right)^{2} / D(v)=D\left(q_{i}\right) / Q^{2},
$$

where now $q_{i}=v_{, i}$.

If we compare Eqs. (1.1) and (2.1), we see that we can include both in a single definition, if we define the resistance in general as the ratio of the average inlet-outlet temperature difference to the total input heat power. This is the definition which we shall adopt. It is particularly apt because it admits immediately of a simple physical interpretation and because, at least in the two cases considered so far, it may be expressed in terms of the Dirichlet integral which is easily bounded.

3. The Robin-Neumann problem. We now consider a heat flow problem which we regard as canonical and from which the two preceding problems may be derived as special cases. We again seek a harmonic function $w$, now satisfying the boundary conditions 


$$
w_{, i} n_{i}=\left\{\begin{array}{lll}
h_{1}\left(w_{1}-w\right), & \text { on } & S_{1}, \\
0, & \text { on } & S_{a}, \\
h_{0}\left(w_{0}-w\right), & \text { on } & S_{0},
\end{array}\right.
$$

where $w_{1}$ and $w_{0}$ are constant inlet and outlet ambient temperatures and $h_{1}$ and $h_{0}$ are positive, constant heat transfer coefficients.

These boundary conditions approximate a wide variety of modes of surface heat transfer, including radiation, convection, and contact resistance. For large $h$ the boundary conditions approach the Dirichlet conditions satisfied by $u$ and for small $h$ (with some reservations as to the character of the limiting process) the Neumann conditions satisfied by $v$. We therefore expect that in this case the resistance will have a value intermediate between $R(u)$ and $R(v)$, namely

$$
R(u) \leq R(w) \leq R(v),
$$

where

$$
R(w)=\left(\langle w\rangle_{1}-\langle w\rangle_{0}\right) / Q(w),
$$

according to our general definition of resistance. In the next section we obtain upper and lower bounds on $R(u)$ and $R(v)$ which we then use to derive the inequality (3.1).

4. Upper and lower bounds on resistance. The problem of bounding the resistances $R(u)$ and $R(v)$ is equivalent to bounding the corresponding Dirichlet integrals. Such bounds may be obtained in a variety of ways (see, e.g., [2], [3], [4]). For our purposes, the most direct approach is the use of Schwarz's inequality, as suggested by Diaz and Weinstein [1]. We first state the results and the conditions on the bounding functions and then give a brief derivation of one of them.

We have

$$
\begin{gathered}
\left(u_{1}-u_{0}\right)^{2} / D(f) \leq R(u) \leq D\left(p_{i}\right) / Q^{2}\left(p_{i}\right), \\
\left(\langle f\rangle_{1}-\langle f\rangle_{0}\right)^{2} / D(f) \leq R(v) \leq D\left(p_{i}\right) / Q^{2},
\end{gathered}
$$

where, as before,

$$
\begin{array}{rlrl}
D(f) & =\int_{V} f_{, i} f_{, i} d V, & D\left(p_{i}\right)=\int_{V} p_{i} p_{i} d V, \\
Q\left(p_{i}\right) & =\int_{S_{1}} p_{i} n_{i} d S, & & \\
\langle f\rangle_{1} & =S_{1}^{-1} \int_{S_{i}} f d S, & \langle f\rangle_{0} & =S_{0}^{-1} \int_{S_{0}} f d S .
\end{array}
$$

In these inequalities $f$ is any non-constant scalar function, continuous on $V+S$, piecewise continuously differentiable in $V$, and satisfying any boundary conditions of Dirichlet type given on $S$. Thus in (4.1)

$$
f=\left\{\begin{array}{lll}
u_{1}, & \text { on } & S_{1} \\
u_{0}, & \text { on } & S_{0}
\end{array}\right.
$$

while in (4.2) it is unrestricted. The vector $p_{i}$ is any non-zero, solenoidal vector function, having a continuous normal component across any surface in $V$, piecewise continuous derivatives in $V$, and such that its normal component satisfies any given Neumann 
conditions on $S$. Thus in both (4.1) and (4.2)

$$
\begin{aligned}
& p_{i, i}=0, \text { in } V, \\
& p_{i} n_{i}=0, \text { on } S_{a},
\end{aligned}
$$

while, in addition in (5.2),

$$
p_{i} n_{i}=\left\{\begin{array}{lll}
Q / S_{1}, & \text { on } & S_{1} \\
-Q / S_{0}, & \text { on } & S_{0}
\end{array} .\right.
$$

As a sample, let us derive the upper bound on $R(u)$. For two arbitrary vector functions, $p_{i}$ and $q_{i}$, Schwarz's inequality takes the form

$$
\left[D\left(p_{i}, q_{i}\right)\right]^{2} \leq D\left(p_{i}\right) D\left(q_{i}\right)
$$

where, using obvious notation,

$$
D\left(p_{i}, q_{i}\right)=\int_{V} p_{i} q_{i} d V
$$

If we set $q_{i}=u_{, i}$, this becomes

$$
\left[D\left(p_{i}, u_{, i}\right)\right]^{2} \leq D\left(p_{i}\right) D(u) .
$$

Green's identity gives

$$
D\left(p_{i}, u_{, i}\right)=\int_{S} u p_{i} n_{i} d S=\left(u_{1}-u_{0}\right) Q\left(p_{i}\right),
$$

using the boundary conditions on $p_{i}$ and $u$. Thus

$$
\left(u_{1}-u_{0}\right)^{2} / D(u) \leq D\left(p_{i}\right) / Q^{2}\left(p_{i}\right),
$$

which is the required inequality. The remaining bounds follow in so similar a fashion that there is no need to reproduce their derivation here.

Now note that we may set $p_{i}=w_{, i}$ in the upper bound on $R(u)$ and $f=w$ in the lower bound on $R(v)$, giving

$$
\begin{aligned}
R(u) & \leq R(w) D(w) / Q(w)\left(\langle w\rangle_{1}-\langle w\rangle_{0}\right), \\
R(v) & \geq R(w) Q(w)\left(\langle w\rangle_{1}-\langle w\rangle_{0}\right) / D(w) .
\end{aligned}
$$

We now show that

$$
D(w) \leq Q(w)\left(\langle w\rangle_{1}-\langle w\rangle_{0}\right)
$$

from which the inequality

$$
R(u) \leq R(w) \leq R(v)
$$

follows.

Green's identity gives

$$
\begin{aligned}
Q(w) & =\int_{S_{2}} w_{, i} n_{i} d S=h_{1} S_{1}\left(w_{1}-\langle w\rangle_{1}\right) \\
& =-\int_{S_{0}} w_{, i} n_{i} d S=-h_{0} S_{0}\left(w_{0}-\langle w\rangle_{0}\right),
\end{aligned}
$$


and

$$
D(w)=h_{1} S_{1} w_{1}\langle w\rangle_{1}+h_{0} S_{0} w_{0}\langle w\rangle_{0}-h_{1} \int_{S_{1}} w^{2} d S-h_{0} \int_{S_{\circ}} w^{2} d S .
$$

Schwarz's inequality gives

$$
S_{1} \int_{S_{2}} w^{2} d S \geq\left[\int_{S_{1}} w d S\right]^{2}
$$

or

$$
h_{1} \int_{S_{1}} w^{2} d S \geq h_{1} S_{1}\langle w\rangle_{1}^{2}
$$

and similarly for the integral over $S_{0}$, so that

$$
D(w) \leq h_{1} S_{1}\langle w\rangle_{1}\left(w_{1}-\langle w\rangle_{1}\right)+h_{0} S_{0}\langle w\rangle_{0}\left(w_{0}-\langle w\rangle_{0}\right)=Q(w)\left(\langle w\rangle_{1}-\langle w\rangle_{0}\right),
$$

which is the desired inequality.

5. Conclusion. We have now shown that the thermal resistance, i.e., the average inlet-outlet temperature difference for unit input heat power, in the case of the RobinNeumann problem is bounded below by the resistance $R(u)$ for constant inlet and outlet temperatures and above by the resistance $R(v)$ for constant inlet and outlet heat flux. We have also shown how upper and lower bounds on $R(u)$ and $R(v)$ may be obtained. Thus we have a method for estimating the temperature rise which is relatively independent of the specific nature of the boundary conditions. It is felt that this method of obtaining broad bounds on temperature rise in many cases is much more practical than, for example, the calculation of an exact solution. It is particularly suited to the kind of problem encountered, for example, in preliminary design work, where one knows little about the specific nature of the heat flow and where one may want to evaluate the effect of changes in many design parameters.

From the mathematical point of view, the foregoing results are quite trivial. It is interesting to note, however, that they provide another illustration of the complementary nature of the Dirichlet and Neumann conditions. According to the Dirichlet principle, among all functions having given constant values on inlet and outlet, the harmonic function $u$ makes the Dirichlet integral $D(u)$ smallest. If we relax this condition and require only that the admissible functions have a given difference of average values between inlet and outlet, the Dirichlet integral is minimized by the harmonic function $v$. The first result gives the lower bound on $R(u)$, the second the lower bound on $R(v)$. The upper bounds may be similarly described, using the Thomson principle concerning solenoidal vectors.

\section{BIBLIOGRAPHY}

1. J. B. Diaz and A. Weinstein, Schwarz's inequality and the methods of Rayleigh-Ritz and Trefftz, J. Math. Phys. 27, 133 (1948)

2. P. Cooperman, An extension of the method of Trefftz for finding local bounds on the solutions of boundary value problems, and on thier derivatives, Quart Appl. Math. 10, 359 (1953)

3. G. Polya and G. Szegö, Isoperimetric inequalities in mathematical physics, Princeton University Press, 1951

4. J. L. Synge, The hypercircle in mathematical physics, Cambridge University Press, 1957 